\title{
La peste des petits ruminants au Sénégal Données nouvelles
}

\author{
par P. BOURDIN $\left({ }^{*}\right)$ et M. P. DOUTRE $\left({ }^{* *}\right)$
}

\begin{abstract}
RESUMÉ
Les auteurs rapportent l'apparition d'une importante épizootie de peste des petıts rumınants au Sénégal lors de la saison des pluies 1974. Depuis cette date, la maladie provoque des pertes considérables dans différentes régions du pays. L'épidémiologie et l'aspect clinique, les techniques de virologie mises en œuvre dans le dıagnostic de routine, les complicatıons d'origine microbienne sont décrites. En raison de la valeur commercıale élevée des ovins et des caprins, la nécessité d'une action prophylactıque efficace, menée en temps voulu (fin de saison sèche) est soulıgnée. Le diagnostic différentiel est facilité par le recours au laboratoire.
\end{abstract}

Signalée pour la première fois au Sénégal en 1955 par MORNET, ORUE, GILBERT, THIERY et SOW (10), la peste des petits ruminants (PPR) était déjà décrite, en 1940, en Côte-d'Ivoire, par GARGADENNEC et LALANNE (8), et en 1941, au Dahomey, par CATHOU (6), chez la chèvre naine dite des lagunes. Au Nigeria méridional, WHITNEY, SCOTT et HJLL, en 1967 (19) signalent une affection semblable de la même espèce qu'ils dénomment " Kata » (stomatite et entérite de la chèvre). Par la suite, en 1970, ROWLAND et BOURDIN (17) et, en 1971, ROWLAND, SCOTT, RAMACHANDRAN et HILL (18) conviennent que « Kata » et " $P P R$ » relèvent du même virus. Au Nigeria du Nord, ZWART et ROW (20), en 1966, puis au Tchad, PROVOST, MAURICE et BORREDON (13, 14), après enquêtes chez les ovins et caprins sahéliens, estiment que la maladie existe mais qu'elle évolue sous une forme inapparente.

Au Sénégal, l'affection demeure à l'état

(*) Chef du Service de Virologie du Laboratorre National de l'Elevage et de Recherches Vétérinaires (I. S. R. A.), B. P. 2057, Dakar, Sénégal.

(**) Chef du Service de Bactériologie du Laboratoire National de l'Elevage et de Recherches Vétérinaires (I. S. R. A.). enzootique. Elle touche essentiellement les chèvres et plus rarement les moutons $(4,5)$. Elle frappe surtout les jeunes de 2 à 18 mois et les pertes varient selon les années. Son existence est certainement beaucoup plus ancienne et le diagnostic de pasteurellose ovine ou caprine, avancé fréquemment dans les rapports des Services de l'Élevage doit masquer souvent une étiologie primitive à virus PPR.

Entre 1969 et 1974, plusieurs foyers sont étudiés (Thiès, Linguère, Diourbel, Dahra), le diagnostic reposant sur les commémoratifs, les signes cliniques, l'étude anatomo-pathologique et les examens de laboratoire: précipitodiffusion en milieu gélosé, isolement du virus sur cellules d'épithélium rénal de fotus de mouton de première explantation à partir du sang, de la rate, du mucus nasal et des ganglions trachéo-bronchiques. De plus, fréquemment, des pneumonies et des pleuropneumonies à Pasteurella multocida sont observées.

Dans le passé, en raison de l'extrême localisation des cas enregistrés, du petit nombre de sujets touchés et de leur valeur marchande réduite, l'attention des autorités vétérinaires n'était retenue que d'une façon modérée, et seuls les services de Virologie et de Bactério- 
logie du Laboratoire de Dakar consacraient une faible partie de leur activité à une maladie dont l'importance économique demeurait des plus restreintes.

Au cours de la saison des pluies 1974, le nombre des foyers s'est soudainement accru et dès lors, le terme d'épizootie pouvait être retenu pour caractériser une cause de mortalité en pleine extension. Le Ferlo, la vallée du fleuve Sénégal (Matam, etc...), le Sine-Saloum (Unités expérimentales de l'I. R. A. T.), la région du Cap Vert, etc... voient leurs troupeaux de petits ruminants décimés, parfois dans leur totalité, les jeunes payant le plus lourd tribut... La fin de la saison sèche amène une certaine rémission, mais avec la venue des pluies 1975, une nouvelle flambée, dont les effets peuvent encore être constatés, se manıfeste... Il semble raisonnable de croire que la PPR ne se cantonne pas à la rive gauche du fleuve Sénégal et qu'au moins la partie méridionale de la Mauritanie est à inclure dans la zone géographique atteinte par la maladie. Au Sud, la Gambie est officiellement concernée.

La présente note se propose de relater les observations effectuées et les résultats des analyses accomplies au Laboratoire de Dakar. En raison des années de sécheresse passée, le Sénégal est importateur de moutons. Sur le marché de la viande, le prix des animaux s'est considérablement accru. Des études sont en cours pour améliorer le rendement de la production, des techniques d'embouche sont mises au point. Aussi, est-il souhaitable que, sur le plan de la pathologie, la PPR retienne l'attention et qu'elle soit combattue avec efficacité et persévérance comme le furent les grandes affections, peste et péripneumonie, anciennes entraves au développement du cheptel bovin. D'autant que la possibililité d'une prophylaxie médicale valable est démontrée : au Dahomey, depuis 1969 (2), et au Sénégal, depuis 1970 (4).

L'élevage des caprins, en zone sahélienne, fait l'objet d'âpres controverses... Accusé d'avoir une action néfaste directe (broutage) et indirecte (émondage abusif effectué par les bergers) sur la strate ligneuse, la chèvre demeure pour d'autres, sinon "la vache du pauvre " tout au moins celle des femmes et des enfants (dans le Sine-Saloum, 80 p. 100 des propriétaires de caprins sont des femmes). Constituant un apport protéique et laitier non négligeable, l'espèce mérite protection.

\section{ÉPIDÉMIOLOGIE}

Les principaux foyers apparaissent dans le nord du pays pendant la saison des pluies, l'extension vers le sud et vers l'est s'effectue par la suite, grâce à la transhumance. Le froid relatif des mois d'hiver exerce une action favorisante certaine, les précipitations d'été jouent sans doute le même rôle en refroidissant les animaux.

La transmission se fait par contact et la voie de pénétration est principalement la muqueuse nasale (3).

\section{SYMPTOMATOLOGIE}

La maladie est décrite en détails par MORNET et collab. (10). Il convient de distinguer une forme suraiguë et une forme aiguë.

Dans la forme suraiguë, l'évolution dure entre 4 et 5 jours, l'hyperthermie $\left(40,41^{\circ}\right)$, l'anorexie, l'aspect frileux, le jetage nasal et la diarrhée constituent les seuls signes visibles. La mort est en général brutale.

Dans la forme aiguë, l'évolution se poursuit pendant 8 à 10 jours. On retrouve la symptomatologie précédente, mais souvent la dypsnée et la toux traduisent l'atteinte de l'appareil respiratoire avec pneumonie ou pleuropneumonie due aux complications bactériennes. L'apparition d'ulcères au niveau des gencives est possible mais rare. De même, peut-on noter l'existence d'un enduit pultacé sur la langue. L'amaigrissement survient puis la mort. Des avortements sont observés chez les femelles.

Dans les formes qui évolueraient sans doute vers la guérison, on enregistre parfois une fausse « parésie » des membres, le dos des malades est voussé. En raison du contexte « maladie des forages $»$, toujours présent dans les esprits, les éleveurs parlent de " gniedio » (maladie des membres), les infirmiers accusent le botulisme... Des feux en raies sont appliqués de part et d'autre de la colonne vertébrale... Sans aucun succès. En fait, l'autopsie de ces sujets révèle que le rumen est dilaté par une masse d'aliments dure et très sèche. L'atonie de la panse entraîne une indigestion par surcharge, d'où la démarche anormale liée à la gêne abdominale. Dans de tels cas, la constipation demeure la règle. 


\section{DIAGNOSTIC VIROLOGIQUE}

Les prélèvements sont soumis à deux tests : l'immunodiffusion en milieu gélosé et l'isolement sur cellules rénales de fœus de mouton.

L'immunodiffusion met en cuvre la technique classique d'Ouchterlony, en boîte de Pétri, appliquée à la peste bovine (intervalle entre les cupules: de 5 à $7 \mathrm{~mm}$ ). Le sérum hyperimmun provient de lapins inoculés avec une suspension du virus vaccinal bovipestique (souche RPOK/BK 70 passages de PLOWRIGHT et FERRIS), cette suspension étant clarifiée par centrifugation. L'antigène est un fragment de rate, de ganglion lymphatique ou de parenchyme pulmonaire offrant des lésions de pneumonie au stade d'hépatisation rouge. Les lignes de précipitation apparaissent en 24 à 48 heures à la température de $20^{\circ} \mathrm{C}$.

En ce qui concerne l'isolement, les cultures cellulaires sont utilisées de préférence à l'inoculation à la chèvre, bien que l'extrême sensibilité de l'espèce naine des lagunes soit reconnue. En 1962, GILBERT et MONNIER (9) ont mis en évidence la replication du virus sur cellules rénales de fœtus de mouton. Depuis 1969 (2), le virus PPR est isolé plus ou moins régulièrement à partir de sujets malades. Mais il est impératif de disposer d'organes frais, bien conservés sous glace, issus d'animaux en hyperthermie marquée et malades depuis 5 jours au maximum. Sont utilisés : le mucus nasal, le sang hépariné, les ganglions lymphatiques, la rate et le parenchyme pulmonaire pris au niveau d'une lésion de pneumonie au stade d'hépatisation rouge.

Le sang hépariné demeure une heure en contact avec le tapis cellulaire. Le mucus nasal, recueilli par écouvillonnage, est mis à diffuser dans une solution de Hanks-Laye antibiosupplémentée. Cette solution reste en contact avec le tapis pendant une heure.

Les ganglions lymphatiques, la rate, le parenchyme pulmonaire sont découpés puis déposés dans une solution de Hanks-Laye, dans le rapport de 1 partie d'organe pour 20 de solution.

Après broyage et centrifugation, on inocule le surnageant. Le contact avec le tapis dure une heure, puis les cellules sont rincées pour éviter les effets toxiques. Le milieu d'entretien classiquement employé est la solution de HanksLaye à 3 p. 100 de sérum de veau. Les lésions cellulaires apparaissent entre 8 et 15 jours selon les souches de virus.

\section{COMPLICATIONS BACTÉRIENNES}

Elles ont été étudiées au niveau des appareils respiratoire et digestif :

\section{Appareil respiratoire}

$\mathrm{La}$ majorité des résultats rapportés cidessous résulte d'ensemencements accomplis sur le terrain, avec le matériel nécessaire, à partir de lésions du parenchyme pulmonaire ou de liquide pleural. Ensuite, des prélèvements sont rapportés au laboratoire, sous froid, pour la recherche éventuelle de mycoplasmes. Ont été ainsi identifiés :

- Bactéries Gram + : Streptocoques, Pneumocoques.

- Bactéries Gram - : Pasteurella multocida, type A. 3 (indole + , betagalactosidase -) (*), Pasteurella hemolytica (indole beta-galactosidase $\rightarrow$ (12), deux souches offrant les caractères généraux de $P$. hemolytica (indole -), mais betagalactosidase — ont été obtenues. L'étude de la constitution antigénique de ces 2 souches par électrophorèse en gel d'acrylamide effectuée par PERREAU (I. E. M. V. T.) a montré qu'elles s'apparentaient par leur schéma de structure protéique au genre Pasteurella. Elles doivent donc être momentanément classées comme Pasteurella sp. Ces Pasteurella atypiques sont certainement à ranger dans le groupe des Pasteurella likeorganisms évoqué par G. R. CARTER (6). L'isolement de souches nouvelles révèle une systématique plus complexe du genre Pasteurella.

Il est à noter la mise en évidence de $P$. hemobytica chez des moutons sains sacrifiés à l'abattoir de Dakar, au cours d'une brève enquête destinée à confirmer le portage chronique (écouvillonnage de la muqueuse trachéale et ensemencement sur gélose sérum).

- Autres germes Gram - isolés : Salmonella ( $S$. typhimurium d'un poumon de chèvre), Klebsiella, Escherichia coli, Serratia, Pseudomonas aeruginosa.

(*) Le sérotypage des souches de $P$. mulsocida a été réalisé par le Service de Bactériologie de l'I. E. M. V.T. (P. PERREAU). 
En 1974-1975, les lésions de pneumonie et de pleuro-pneumonie et les isolements de germes appartenant au genre Pasteurella ont manifesté une plus grande fréquence. En 1976, l'appareil respiratoire est bien souvent indemne, et les lésions, lorsqu'elles existent, montrent à l'isolement des germes Gram + et des entérobactéries.

Les atteintes étendues du parenchyme pulmonaire, dues à l'action bactérienne, favorisent la mort de l'animal dans les formes aiguës de la maladie.

Depuis 1974, aucun mycoplasme n'a été isolé des prélèvements rapportés au laboratoire (Bacto PPLO-agar additionné de 20 p. 100 de sérum de cheval décomplémenté).

\section{Appareil digestif}

La recherche de Salmonella (après enrichissement en bouillon sélénite) dans les excréments n'a donné aucun résultat. Par contre, la présence quasi constante de Pseudomonas aeruginosa parmi la flore bactérienne (Gram +, entérobactéries diverses, anaérobies, etc...) a été constatée sans qu'une importance particulière y ait été portée.

\section{DISCUSSION}

La PPR demeure actuellement la maladie virale la plus meurtrière du Sénégal. L'épizootie débuta pendant la saison des pluies 1974 et, depuis cette date, on note lextension de l'affection.

Jusqu'alors, en région sahélienne, la PPR sévissait à l'état sporadique avec des poussées de recrudescence $(1961,1965,1970)$. La faible valeur économique des petits ruminants, et tout particulièrement des caprins, limitait la prophylaxie médicale à des actions ponctuelles conduites par le Laboratoire.

La diminution du troupeau bovin, conséquence des années de sécheresse, a valorisé les petits ruminants, d'où la nécessité, pour les agents des Services de l'Élevage, de mieux connaître la maladie et sa prévention.

Le concept de " pasteurellose », très ancien, a retardé l'identification sur le terrain de la PPR. Les symptômes respiratoires, souvent présents, entretiennent la confusion. La pasteurellose n'est qu'une complication possible mais non absolue.
Le clinicien doit suspecter la PPR lorsqu'il note une maladie extrêmement contagieuse, à mortalité élevée, survenant surtout chez les jeunes, avec comme signes dominants : l'hyperthermie marquée, le jetage, le larmoiement, l'éternuement, puis la toux, la dypsnée et enfin l'entérite aiguë, rapidement déshydratante, qui précède de peu la mort. Le test de précipitodiffusion, exécuté au laboratoire, avec des prélèvements bien conservés (rate, ganglions mésentériques, poumon) permet la rapide confirmation du diagnostic clinique.

Le diagnostic différentiel doit élıminer:

- l'ecthyma contagieux : où dominent les lésions péribuccales croûteuses ;

- la variole caprine : caractérisée par des pustules au niveau de l'abdomen;

- la clavelée : spécifique au mouton, avec comme signe pathognomonique l'existence de nodules sous-cutanés, fermes à la palpation, au niveau de la tête et de la partie antérieure du corps;

- la rickettsiose ovine : provoquée par Rickettsia ovina, transmise par les tiques sahéliennes du genre Hyalomma et Boophilus. Elle frappe, dans un troupeau, les plus beaux sujets ou évolue sous forme subaiguë en 8 à 10 jours. Les malades présentent de l'asthénie, de l'anorexie, de la constipation, de l'amaigrissement, des signes nerveux épisodiques et finalement succombent. A l'autopsie, ascite, hydrothorax et hydropéricarde sont observés. L'agent causal est mis en évidence dans les monocytes sur frottis de sang périphérique. Cette rickettsiose a été constatée cette année au Sénégal (3). La Heart Water (Cowdria ruminantium) sévit dans les zones à Amblyomma et offre des signes cliniques et nécropsiques semblables. Seul l'examen de frottis de cerveau et d'endoveine permet la différenciation;

- la pleuro-pneumonie des chèvres à $\mathrm{Myco-}$ plasma mycoides caprae, inconnue jusqu'alors au Sénégal ;

- la coccidiose et les helminthoses qui aggravent le pronostic.

BOURDIN, LAURENT et RIOCHE (2, 4, 5) ont réalisé la prophylaxie médicale de la PPR au Dahomey (20 000 animaux) et au Sénégal (essais de laboratoires, puis 6000 têtes des troupeaux des Régions de Thiès et de Diourbel).

Le vaccin est celui utilisé dans la lutte contre la peste bovine (PB) (culture sur épithélium 
rénal de factus de veau de la souche de PLOWRIGHT et FERRIS au $70^{\circ}$ passage). Les conditions d'emploi demeurent presque identiques : $1 \mathrm{ml}$ de la suspension, reconstituée en eau distillée ou sérum physiologique, injecté par voie sous-cutanée, à tous les animaux âgés de plus de deux mois, en fin de saison sèche, époque de rémission de la maladie.

La vaccination pratiquée dans les foyers, d'individus en incubation, apparaît décevante. L'immunité nécessite 5 jours pour s'installer.

Le choix du virus vaccinal $P B$ repose sur l'existence des rapports antigéniques étroits des virus PPR et PB, établis en 1956 (10), puis confirmés en 1962 et 1969 (9 et 2).

Le virus PPR est-il un virus PB adapté aux petits ruminants et dépourvu de pouvoir pathogène pour les bovins? Tous les essais de transmission tentés à Dakar $(10,2)$ montrent que zébus et taurins réagissent très faiblement à l'inoculation du virus PPR. Absent du mucus nasal et des selles (2), le virus se rencontre uniquement dans le sang 48 heures après introduction sous la peau.
Des chercheurs indiens, NARAYANASWAMY et RAMANI, en 1973 (11) puis RAMANI, CHARLES et RAMACHANDRAN, en 1974 (15) rapportent de graves foyers de «peste bovine » chez les petits ruminants du district de Karnataka et isolent un virus beaucoup plus pathogène pour le mouton que pour le zébu et le buffle. RAO, DEVI, RAMACHANDRAN et SCOTT, en 1974 (16), dans le district d'Andhara Pradesh, mettent en évidence, à partir d'ovins malades, une souche à virulence élevée pour le mouton et moyenne pour le buffle. Ces mêmes auteurs, ainsi que BANSAL et JOSHI (1), soulignent les bons résultats obtenus par l'utilisation du vaccin $\mathrm{PB}$ de culture cellulaire dans le contrôle de l'épizootie sévissant chez les petits ruminants.

Si l'on compare les travaux des auteurs africains et indiens, l'identité des deux affections étudiées apparaît, mais la virulence des souches ouest-africaines pour les bovins semble plus faible que celle des souches indiennes. Ce fait illustre une fois de plus la variabilité bien connue du virus de la peste bovine.

\section{SUMMARY}

Pseudo-rinderpest of small ruminants in Senegal. New data.

The authors report an important epizooty of peuso-rinderpest of small ruminants which broke out in Senegal during the 1974 rainy season. Since the losses caused by the disease are heavy. Epidemiology and clinical aspects virological methods employed in the routıne diagnosis, bacteriological complications are described. On account of the high commercial value of sheep and goats, an efficient prophylactic action must be carried out at the right time of the year (end of the dry season). Differential diagnosis is made easier with the help of the laboratory.

\section{RESUMEN}

\section{La peste de los pequeños rumiantes en Senegal. Nuevos datas}

Los autores notan la aparición de una importante epizootıa de peste de los pequeños rumiantes en Senegal durante la estación de las lluvias de 1974. Desde entonces, la enfermedad está provocando perdidas importantes en varias regiones del pais. Se describen la epidemiologia y el aspecto clinico, las tecnicas de virologia utilizadas para el diagnóstico, las complicaciones de origen microbiano. A causa del valor comercial elevado de los ovinos y de los cabrunos se necesita una accion profiláctica eficaz, efectuada al fin de la estación seca. El recurso al laboratorio facilita el diagnóstico diferencial.

\section{BIBLIOGRAPHIE}

1. BANSAL (R. P.) et JOSHI (R. C.). Studies on use of tissue culture rinderpest vaccine among sheep and goats. Ind. vet. J., 1974, 51 (9/10): 631-634.

2. BOURDIN (P.), RIOCHE (M.) et LAURENT (A.). Etude de la peste des petits ruminants. Rapport final, convention FAC 45/C/66/G, 1969, projet $n^{\circ} 163$, Paris, I. E. M. V. T., 59 p.
3. BOURDIN (P.). Observation non publiée, 1970, 1976.

4. BOURDIN (P.) et LAURENT (A.). Elat actuel des recherches sur la prophylaxie médicale de la peste des petits ruminants. XL' session générale du Comité de l'O. l. E., Paris, mai 1972, rapport 200. 
5. BOURDIN (P.). La peste des petuts ruminants (P. P. R.) et sa prophylaxie au Sénégal et en Afrique de 1'Ouest. Rev. Elev. Méd. vét. Pay's trop., 1973, 36 (4) : 71-74.

6. CARTER (G. R.). Diagnostic procedures in veterinary microbiology $2^{\circ}$ édition. Springfield, Illinois U. S. A., Charles C. Thomas, 1973.

7. CATHOU (P.). Rapport annuel du Service de l'Elevage du Dahomey, 1941.

8. GARGADENNEC (L.) et LALANNE (A.). La peste des petits ruminants. Bull. Serv, zoot. A. O. F., $1942,5: 16$.

9. GILBERT (Y.) et MONNIER (J.). Adaptation du virus de la peste des petits ruminants aux cultures cellula1res. Rev. Elev. Méd. vét. Pays trop., 1962, 15 (4) : 32L-335.

10. MORNET (P.), ORUE (J.), GILBERT (Y.) THIERY (G.) et SOW (M.). La peste des petits ruminants en Afrique Occidentale Française et ses rapports avec la peste bovine. Rev. Elev. Méd. vét. Pays trop., 1956, 9 (4) : 313-342.

11. NARAYANASWAMY (M.) et RAMANI (K). Prelıminary studies on rinderpest virus from outbreaks in sheep in Mysore state. Ind. yet. J., 1973, 50 (8) : 829-832.

12. PEGRAM (R. G.). Serological types of Pasteurella haemolytica isolates from sheep and goats in the Somali Democratic Republic. Trop. anim. HIth Prod., 1974, 6 (4) : 189-191.
13. PROVOST (A.), MAURICE (Y.) et BORREDON (C.). La peste des petits ruminants existe-telle en Afrique centrale ? XL $\mathrm{X}^{*}$ Session générale du comité de l'O. I. E. Paris, mai 1972, rapport 202.

14. PROVOST (A.). Rapport annuel, 1974, p. V 27.

15. RAMANI (K.), CHARLES (Y.S.) et RAMACHANDRAN (S.). Further studies on rinderpest virus of sheep origin. Ind. vet. J., 1974, 51 (2) : 129-138.

16. RAO (M.), DEVI (T. 1.), RAMACHANDRAN (S.) et SCOTT (G. R.). Rinderpest in sheep in Andhra Pradesh and its control by vaccination. Ind. vet. $J$., 1974, 51 (6) : 439-450.

17. ROWLAND (A. C) et BOURDIN (P.) The histological relationship between «Peste des petits rummants " and "Kata » in West Africa. Rev. Elev. Méd. vét. Pays trop., 1970, 23 (3) : 301-307.

18. ROWLAND (A. C.), SCOTT (G. R.), RAMACHANDRAN (S.) et HILL (D. H.). A comparative study of «Peste des petits ruminants» and "Kata» in West African dwarf goats. Trop. anim. Hith Prod., 1971, 3 (4) : 241-245.

19. WHITNEY (J. C.), SCOTT (G. R.) et HILL (D. H.). The pathology of an erosive stomatitis and enteritis of goats in Southern Nigeria. Bull. epiz. Dis. Afr., 1967, 15 (1): 31-41.

20. ZWART (D.) et ROWE (L. W.). The occurence of rinderpest antibodies in the sera of sheep and goats in Northern Nigeria. Res. vet. Sci., 1966, 7 (4) : 504-511. 